\title{
Kernos
}

Revue internationale et pluridisciplinaire de religion grecque antique

12| 1999

Varia

\section{Apollon Lykeios dans la tragédie : dieu protecteur, dieu tueur, " dieu de l'initiation »}

\section{Claire-Françoise de Roguin}

\section{(2) OpenEdition \\ Journals}

Édition électronique

URL : http://journals.openedition.org/kernos/713

DOI : $10.4000 /$ kernos.713

ISSN : 2034-7871

Éditeur

Centre international d'étude de la religion grecque antique

Édition imprimée

Date de publication : 1 janvier 1999

Pagination : 99-123

ISSN : 0776-3824

Référence électronique

Claire-Françoise de Roguin, « Apollon Lykeios dans la tragédie : dieu protecteur, dieu tueur, « dieu de l'initiation » », Kernos [En ligne], 12 | 1999, mis en ligne le 13 avril 2011, consulté le 01 mai 2019. URL : http://journals.openedition.org/kernos/713; DOI : 10.4000/kernos.713 


\section{Apollon Lykeios dans la tragédie : dieu protecteur, dieu tueur, « dieu de l'initiation »}

L'épithète Lykeios, désignant Apollon, se trouve, d'une part, dans des inscriptions, sur des monnaies et comme nom de mois, dans diverses régions de Grèce et d'Asie mineure, et témoigne ainsi d'une certaine importance du culte d'Apollon Lykeios ${ }^{1}$. D'autre part, le nom d'Apollon Lykeios apparaît dans divers textes, en particulier dans plusieurs tragédies d'Eschyle et de Sophocle. Un certain nombre de travaux, anciens et récents, ont été consacrés à Apollon Lykeios; mais aucun d'entre eux n'envisage dans leur ensemble les diverses interventions, dans la tragédie, du dieu désigné par cette épithète. Il semblait donc intéressant d'examiner dans quel sens il faut comprendre l'épithète Lykeios, et quel est le rôle joué par Apollon Lykeios dans les tragédies où son nom intervient.

\section{Apollon Lykeios : le tueur de loups?}

On cherchera d'abord à définir le sens de l'épithète Lykeios à partir de textes autres que les tragédies où elle est mentionnée. Le sens de cette épithète est débattu depuis l'Antiquité ${ }^{2}$. Trois principales interprétations en ont été proposées. Les Anciens, comme la plupart des commentateurs modernes,

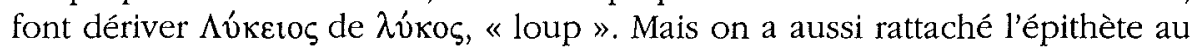

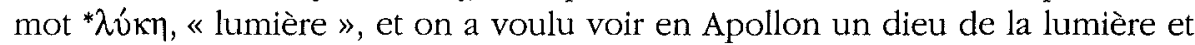
du soleil; mais on considère généralement qu'Apollon n'avait pas, à l'origine, les caractères d'un dieu solaire ${ }^{3}$. Enfin, U, von Wilamowitz, alors qu'il cherchait à imposer l'idée qu'Apollon avait son origine en Asie mineure, a d'abord défendu le sens de « Lycien, de Lycie » pour cette épithète, mais il est revenu

1 Les lieux de culte d'Apollon Lykeios et les documents qui en témoignent sont passés en revue par F. Graf, Nordioniscbe Kulte, Rom, 1985, p. 219-226.

2 Cf. K. WERNICKE, in RE, II 1 (1895), col. 58-60; M.P. NILsSON, Gescbicbte der griecbiscben Religion, Bd. 1, München, 1955, p. 536-538; W. RICHTER, in RE, Suppl. XV (1978), col. 975-977. Les différentes hypothèses ont été discutées en dernier lieu par Graf, op. cit. (n. 1), p. 220-221, avec de nombreuses références anciennes et modernes.

3 WeRNICKE, loc. cit. (n. 2), col. 59; GraF, op. cit. (n. 1), p. 220. 
plus tard sur cette interprétation ${ }^{4}$. W. Burkert a montré cependant que l'épi-

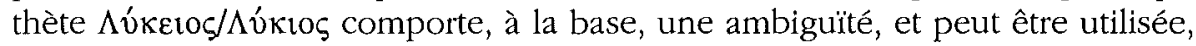
selon les cas, pour mettre en évidence les relations d'Apollon soit avec les loups, soit avec la Lycie $^{5}$. On verra plus loin une autre manifestation de cette ambiguité, où semblent entrer en jeu à la fois le loup, la lumière et la Lycie 6 .

Malgré cette restriction, la dérivation de Lykeios à partir du nom du loup est considérée aujourd'hui comme la plus importante. Toutefois, le sens précis de l'épithète, et les fonctions d'Apollon lorsqu'il est désigné par elle, ne sont pas clairement définis. Pour les préciser, il faut se tourner vers les textes qui nous renseignent sur les liens qu'Apollon entretenait avec le loup : il s'agit de légendes au sujet d'Apollon Lykeios et de divers textes qui évoquent Apollon et le loup ${ }^{7}$. Ces documents nous montreront que l'épithète peut avoir plusieurs sens.

Parmi les légendes qui parlent d'Apollon et du loup, il y a d'abord ce récit de Pausanias, à propos de Sicyone ${ }^{8}:$ "Près de là se trouve un sanctuaire d'Apollon Lykios $(.$.$) . Alors que des loups s'attaquaient si souvent à leurs$ troupeaux que les gens de Sicyone n'en tiraient plus aucun profit, le dieu leur indiqua un endroit où se trouvait un tronc d'arbre desséché, et leur prescrivit de faire manger aux loups l'écorce de cet arbre, mélangée à de la viande. Dès qu'ils en eurent goûté, les loups furent empoisonnés par l'écorce. Ce tronc se trouvait dans le sanctuaire de Lykios, et même les guides sicyoniens ne savaient pas de quelle espèce d'arbre il s'agissait. »

4 U. Von Wilamowitz-MoEllendorf, Apollon, in Hermes, 38 (1903), p. 575-586, ici 585; Der Glaube der Hellenen, Bd. 2, Darmstadt, 1955² [1931-1932], p. 144; Graf, op. cit.

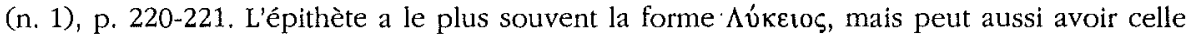
de $\Lambda$ úkıs. On estime parfois que seule la première forme devrait être rattachée au nom du loup, alors que la seconde signifierait «de Lycie» (cf. NilssoN, op. cit. [n. 2], p. 537). Cependant, la forme $\Lambda$ v́kıৎ̧ est clairement mise en rapport avec le loup dans plusieurs documents figurés; c'est aussi la forme des principaux manuscrits des textes de Pausanias, où l'épithète a une relation évidente avec le loup. Il faut donc voir dans cette forme un cas de iotacisme, et non une épithète spécifique (GRAF, op. cit. [n. 1], p. 221, n. 30).

5 W. Burkert, Euenios der Seber von Apollonia und Apollon Lykeios: Mythos jenseits der Texte, in Kemos, 10 (1997), p. 73-81, ici 79.

6 Cf. infra, à propos de SopH., $E d$. Roi, 203.

7 Les mêmes textes sont présentés, d'un point de vue différent, par C. MaINoLdi, L'image du loup et du chien dans la Grèce ancienne d'Homère à Platon, Paris, 1984, p. 22-28; D.E. Gershenson, Apollo the Wolf-god, in Joumal of Indo-European Studies, Monograph 8, 1991, p. 7-16.

8 Paus., II, 9, 7. Sauf mention contraire, les traductions des textes autres que les passages de tragédies sont de l'auteur; les passages de tragédies sont cités dans la traduction, parfois légèrement modifiée, de P. MAZON, Escbyle, tome 1, Paris, 19312 [1920]; Eschyle, tome 2, Paris, 1925; P. Mazon, in A. Dain, P. Mazon, Sopbocle, tome 2, Paris, 1958.

9 La traduction française de cette épithète pose un problème. P. Mazon la traduit généralement par "Lycien », mais cela peut faire penser que l'épithète à un rapport avec la Lycie plutôt qu'avec le loup. On utilisera ici la forme transcrite Lykeios/Lykios (sans toutefois modifier la traduction de Mazon sur ce point). 


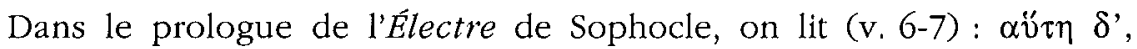

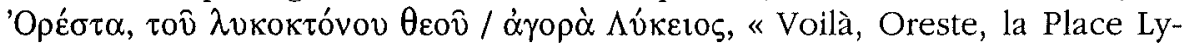
cienne, vouée au dieu tueur de loups ". La scholie au vers 6 explique ainsi le mot $\lambda v$ коктóvo $\varsigma^{10}$ : « Apollon est "tueur de loups", disent les uns, parce qu'il est protecteur des troupeaux et que, lorsqu'il en assure la garde, il tue les loups qui menacent le bétail, et c'est pour cette raison qu'on lui sacrifie des loups à Argos; mais d'autres disent que l'animal lui est consacré, comme la biche l'est à Artémis, et que, pour cette raison, un loup est gravé sur les monnaies d'Argos, comme la chouette sur celles d'Athènes. » Les premières lignes de ce texte s'accordent avec la légende de Sicyone pour présenter Apollon Lykeios comme un dieu tueur de loups, veillant sur les troupeaux. Mais, ajoute le scholiaste, on dit aussi que le loup est consacré à Apollon. On voit donc se dessiner deux aspects contradictoires des relations entre Apollon et le loup.

D'autres textes permettent de mieux définir les rapports entre le dieu et celui qu'on appelle son " animal sacré ». Aristote raconte que Léto se déplaça « de chez les Hyperboréens jusqu'à Délos (...) métamorphosée en louve par crainte d'Héra ${ }^{11}$. Élien dit, pour sa part : «On raconte que le dieu vint au monde alors que Léto était métamorphosée en louve » et que, pour cette raison, "Apollon aime les loups » ${ }^{12}$. Et selon Plutarque également, Apollon, parmi d'autres animaux, aime les loups ${ }^{13}$.

Pausanias, à propos d'Argos, raconte la légende suivante, qui est également rapportée, un peu différemment, par Plutarque ${ }^{14}$ : «Le plus illustre des monuments d'Argos est un sanctuaire d'Apollon Lykios. (...) À l'origine, le temple et la statue de bois ( $\xi o ́ \alpha v o v$ ) furent consacrés par Danaos. (...) Voici pour quelle raison Danaos fonda un sanctuaire d'Apollon Lykios. Lorsqu'il arriva à Argos, il disputa le pouvoir à Gélanor, fils de Sthénélas. Devant le peuple rassemblé, les deux rivaux s'exprimèrent longuement, avec des arguments convaincants, et Gélanor parut avoir plaidé sa cause aussi bien que son adversaire. Mais le peuple, dit-on, remit sa décision au jour suivant. $\mathrm{Au}$ point du jour, un troupeau de bovins paissant devant les murailles fut attaqué par un loup, qui se jeta sur le taureau, le chef du troupeau, et se battit avec lui. Il vint à l'esprit des Argiens de comparer Gélanor au taureau et Danaos au loup : en effet, de même que le loup ne vit pas parmi les hommes, Danaos n'avait pas vécu parmi eux jusqu'alors. Et comme le loup tua le taureau, Danaos obtint le pouvoir. Pensant que le loup avait été conduit vers le troupeau par Apollon, il fonda un sanctuaire d'Apollon Lykios. »

10 P.N. Papageongius, Scbolia in Sopboclis tragoedias vetera, Leipzig, 1888, p. 98-99.

11 Aristr., Hist. an., VI, 35, 580a; traduction : P. Lovis, Aristote, Histoire des animaux, tome 2, Paris, 1968, p. 128.

12 ÉLIEN, Nat. an., X, 26.

13 Plut., Mor., 400a.

14 Paus., II, 19, 3-4; Plut., Pyrb., 32. 
Premièrement, on note qu'Apollon n'est pas ici un «tueur de loups», protecteur des troupeaux; au contraire, il est celui qui envoie un loup pour attaquer un troupeau et tuer le taureau qui en est le chef. Ensuite, le dieu, par l'intermédiaire du loup qu'il envoie pour tuer le taureau, prend le parti de Danaos dans le conflit pour le pouvoir qui l'oppose à Gélanor, et lui permet d'évincer celui-ci. Pour les Argiens, Danaos est celui qui, comme le loup, vient d'un monde étranger, extérieur à leur société, si bien que, dans cette légende, le loup, envoyé d'Apollon, représente aussi Danaos. C'est la volonté du dieu que Danaos prenne le pouvoir, il envoie un loup chargé d'exécuter sa volonté. Le loup tuera le taureau; aux hommes de savoir reconnaître l'intervention divine et d'interpréter correctement le présage. Le loup tueur est l'instrument de la volonté d'Apollon, le représentant de son pouvoir. C'est dans ce sens, peut-être, qu'il faut comprendre, comme le dit la scholie au vers 6 de l'Électre de Sophocle, que le loup est l'animal sacré d'Apollon. En outre, en permettant au « loup » Danaos d'évincer son rival, Apollon Lykeios apparaît à la fois comme son protecteur et comme le garant de son pouvoir ${ }^{15}$.

Selon la même scholie, un loup était gravé sur les monnaies d'Argos. Mais il y avait, à Argos, d'autres effigies de loup. Ainsi, Pausanias, décrivant le sanctuaire d'Apollon Lykios : "Devant le temple se trouve un piédestal orné de sculptures en bas-relief, qui représentent un taureau et un loup en train de combattre, et une jeune fille qui lance une pierre au taureau. On pense que la jeune fille est Artémis. C'est Danaos qui l'a consacré (... $)^{16}$. » De même, Plutarque évoque Pyrrhus « apercevant parmi les nombreux monuments de l'agora un loup et un taureau de bronze qui semblaient engager un combat $\gg{ }^{17}$. Dans ces groupes, le loup peut représenter aussi bien Danaos opposé à Gélanor, que l'animal envoyé par Apollon affrontant le taureau qu'il s'apprête à tuer; mais, si la jeune fille décrite par Pausanias est Artémis, le loup pourrait être Apollon lui-même ${ }^{18}$. Encore un récit de Pausanias, cette fois à propos de Delphes ${ }^{19}$ : «Près du grand autel se trouve une offrande des Delphiens eux-mêmes: il s'agit d'un loup de bronze. On raconte qu'un homme, après avoir volé des trésors appartenant au dieu, s'était caché avec l'or à l'endroit du Parnasse où la forêt est la plus dense. Mais un loup l'attaqua pendant son sommeil et le tua. Après cela, l'animal vint chaque jour dans la ville, où il se mettait à hurler. Quand les Delphiens comprirent que cela ne pouvait se produire sans une intervention divine, ils suivirent le loup; ils trou-

15 À propos du rôle "politique ». d'Apollon Lykeios, cf. $₫ 3$.

PAUS., II, 19, 7.

17 Plut., Pyrrb., 32; traduction: R. Flacelière, E. Chambry, Plutarque, Vies, tome 6, Paris, 1971, p. 73.

18 Selon Servius, in Verg., Aen., IV, 377, où l'on trouve une version différente de la légende de fondation du sanctuaire d'Apollon Lykios à Argos, le loup combattant représenterait Apollon et le taureau Poséidon. La même source indique qu'Apollon prit à deux reprises la forme d'un loup : pour tuer les Telchines et pour s'unir à la nymphe Cyrène.

19 Paus., X, 14, 7. À peu près le même récit chez ÉLien, Nat. an., X, 26; XII, 40. 
vèrent l'or sacré et offrirent un loup de bronze au dieu. » Ici, comme à Argos, le loup se présente comme l'animal d'Apollon, envoyé par le dieu pour tuer le voleur sacrilège et indiquer aux Delphiens l'emplacement du trésor volé. Le loup de bronze représente probablement l'animal sacré du dieu; mais peutêtre représente-t-il le dieu lui-même, puisqu'Élien dit au sujet de cette effigie :

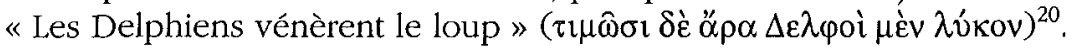

Il est désormais possible de mieux définir les sens que peut prendre l'épithète Lykeios, quand elle accompagne le nom d'Apollon. Apollon Lykeios peut être :

- Apollon « tueur de loups »;

- Apollon « au loup », avec trois sens différents :

Apollon - au loup tueur, c.-à.-d. qui envoie un loup pour tuer, - protecteur du loup ${ }^{21}$,

- qui aime les loups;

- Apollon « loup».

Ces données se déduisent des textes et documents examinés plus haut. Pourtant, beaucoup de commentateurs modernes, se fondant uniquement sur la légende de Sicyone, les vers 6-7 de l'Électre de Sophocle et la scholie au

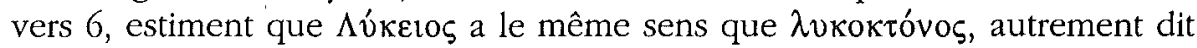
que l'épithète signifie «tueur de loups » et ne peut pas avoir un autre sens; elle serait en rapport avec la fonction de dieu-berger qu'exerce parfois Apollon. Ils admettent toutefois que l'expression «tueur de loups » peut avoir un sens figuré : les « loups » représenteraient ou bien des ennemis, ou bien toutes sortes de fléaux et calamités, contre lesquels Apollon Lykeios est censé protéger, en les écartant ou en les détruisant comme il tue les loups ${ }^{22}$. Il semble y avoir une sorte de réticence à envisager Apollon sous la forme d'un loup, ou même avec des caractères prêtés à cet animal. Quelques auteurs ont noté cependant que le mot Lykeios ne peut pas toujours s'expliquer par le sens de «tueur de loups », au propre ou au figuré. À propos de la légende de Danaos, R.C. Jebb remarque que le loup n'y est pas la victime d'Apollon, mais qu'il est « le symbole d'un pouvoir allié, ou même identifié, à celui d'Apollon

20 Élien, Nat. an., XII, 40. A propos d'autres liens entre Delphes et le loup: W. BURKERT, Homo Necans, Interpretationen altgriechischer Opferriten und Mythen, Berlin, 1972, p. 137-138.

21 Comme le «loup» Danaos ou, comme on le verra plus loin, le « loup » Égisthe ou le « loup » Oreste.

22 Nilsson, op. cit. (n. 2), p. 536; Richter, op. cit. (n. 2), p. 977; R.C. JebB, Sopbocles, The Plays and Fragments, part 1, The Edipus Tyrannus, Cambridge, 1902, p. 38; R.C. Jввв, Sophocles, The Plays and Fragments, part 6, The Electra, Cambridge, 1907, p. 7; p. 205-206; J.C. KamerbeEk, The Plays of Sophocles, Commentaries, part 4, The CEdipus Tyrannus, Leiden, 1967, p. 67; J.C. Kamerbeek, The Plays of Sophocles, Commentaries, part 5, The Electra, Leiden, 1974, p. 22; E. Fraenkel, Aescbylus Agamemnon, vol. 3, Oxford, 1974, p. 581 . 
lui-même » et, à propos de l'histoire du loup de Delphes, que le loup peut être «le symbole du pouvoir violent et destructeur du dieu ». Il note aussi que le loup est un animal aimé d'Apollon. T.G. Tucker admet qu'Apollon peut être tueur de loups; mais, il note, d'autre part, que le dieu aime les loups et qu'enfin, il peut être loup lui-même ${ }^{23}$.

On va voir que, dans la tragédie, Apollon Lykeios peut être "dieu-loup ", « dieu-au-loup » ou « dieu tueur de loups », et qu'il est dangereux de demander sa protection.

\section{Apollon Lykeios dans la tragédie : dieu protecteur ou dieu tueur?}

L'épithète Lykeios apparaît dans trois tragédies d'Eschyle, les Sept contre Thèbes, Agamemnon et les Suppliantes, et dans deux tragédies de Sophocle, CEdipe Roi et Électre. On notera que, dans ces tragédies, l'épithète Lykeios, à une exception près (Soph., $E l$., 7), ne se trouve que dans des prières : prières collectives, où le chœur invoque plusieurs divinités (Esch., Sept, 145; Esch., Suppl., 686; Soph., (Ed. Roi, 203); prières individuelles, où un personnage s'adresse à Apollon Lykeios (Esch., Ag., 1257; Soph., EEd. Roi, 919; Soph., Él., $645,655,1379)$. On examinera d'abord les deux tragédies du cycle thébain (Esch., Sept; Soph., Ed. Roi), puis celles qui traitent du cycle des Atrides (Esch., $A g . ;$ Soph., Él.), enfin les Suppliantes d'Eschyle.

Dans les Sept contre Thèbes, Apollon Lykeios est invoqué dans la parodos (v. 78-180), qui est une prière aux dieux de la cité : les femmes thébaines qui forment le chœur, épouvantées par la nouvelle qu'une attaque argienne se prépare contre la ville, implorent le secours des dieux. Dans cette prière,

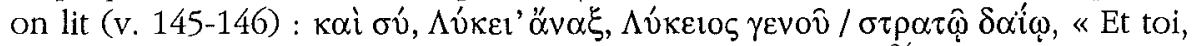
seigneur-loup, montre-toi loup contre l'armée ennemie ${ }^{24}$. Dans cette interprétation, Apollon est considéré lui-même comme un loup et détient le pouvoir destructeur de cet animal. Elle répond bien au texte grec, où l'emploi, à deux reprises, du mot Lykeios sans le nom d'Apollon, semble insister sur le caractère de loup que l'on prête au dieu ${ }^{25}$.

23 Jевв, 1907 (n. 22), p. 206; T.G. Tucker, The Seven against Thebes of Aeschylus, Cambridge, 1908, p. 36. Voir aussi Graf, op. cit. (n. 1), p. 221; MaInoldr, op. cit. (n. 7), p. $27-28$.

24 Traduction d'après A.W. Verrall, The "Seven against Thebes" of Aescbylus, London, 1887, p. 13; Tucker, op. cit. (n. 23), p. 37.

25 U. von Wilamowitz, qui ne conçoit pas Apollon sous la forme d'un loup; mais reconnaît que le loup peut être considéré comme l'animal du dieu, comprend : "Ton nom vient de celui du loup, montre-toi loup contre l'ennemi » (WILAmowitz, 1955 ${ }^{2}$ [1931-1932] (n. 4), p. 143-144). Si, dans la première partie de la phrase, on évite de traiter Apollon comme un « dieu-loup », dans la deuxième, on lui demande quand même de se comporter comme un loup; le sens est finalement à peu près le même que celui retenu ici. La traduction de P. Mazon, en revanche, illustre bien la conception évoquée plus 
Le caractère de loup destructeur d'Apollon Lykeios ressort bien dans la suite de la pièce. À l'issue des combats, en effet (v. $792 \mathrm{~s}$.), on peut croire que la prière prononcée par le chour dans la parodos a été entendue : un messager annonce que la ville est sauvée. Mais, aussitôt, il ajoute qu'Étéocle et Polynice, les deux "rois frères" (v. 804), se sont tués l'un l'autre. C'est Apollon qui est responsable de leur mort : « mais la septième porte, c'est l'auguste dieu Septime, sire Apollon, qui se l'est réservée, pour achever sur la race d'CEdipe le châtiment de Laïos et de ses erreurs anciennes » (v. 800-802); et, plus loin (v. 812-813) : «Et tant pour l'un et l'autre le dieu était égal. C'est lui seul qui détruit la malheureuse race ". Ainsi, l'invocation du chœur au $\Lambda$ v́kєlos $\alpha$ $v \alpha \xi$ semble avoir été exaucée, puisque l'armée argienne a été vaincue : il a bien été le "loup » destructeur de l'ennemi qu'on lui demandait d'être. En revanche, il frappe très durement la cité en étant également le « loup tueur » d'Étéocle et de Polynice. En s'adressant à Apollon Lykeios, le chœur invoque le secours du dieu qui est « directement responsable des malheurs des Labdacides » et qui est donc "dangereux pour la cité » de Thèbes ${ }^{26}$, puisqu'il a juré la perte de la race de ses rois. En réponse à la supplication qui lui est adressée, le dieu frappe mortellement les deux derniers descendants de cette race et provoque ainsi le malheur de la cité ${ }^{27}$. Apollon Lykeios se révèle comme le dieu qui cause la perte de celui qui l'implore.

Dans l'Edipe Roi de Sophocle, Apollon Lykeios est invoqué d'abord dans la parodos (v. 151-215), où le chœur supplie les dieux de délivrer Thèbes du fléau qui la dévaste. Dans la dernière partie du chant, le chœur demande qu'Arès, responsable du fléau, soit chassé de la ville. Il prie Zeus, " maître de l'éclair enflammé », de lâcher sa foudre sur lui (v. 200-202). Puis vient l'invocation suivante (v. 203-208) : «Et toi aussi, dieu Lycien ( $\Lambda$ kel' ’ú $\alpha \xi$ ), je voudrais voir les traits partis de ton arc d'or se disperser, invincibles, pour me secourir, pour me protéger, en même temps que ces flambeaux dont la lueur illumine Artémis, quand elle court, bondissante, à travers les monts de Lycie ( $\Lambda$ úxı'öpe $\alpha$ )». Enfin, le chœur prie Bacchos de venir, « éclairé d'une torche ardente », attaquer Arès (v. 209-215).

Certains commentateurs estiment qu'Apollon Lykeios est invoqué ici comme "tueur de loups ", au sens figuré, c'est-à-dire "destructeur d'ennemis ${ }^{28}$. Selon R.C. Jebb, cependant, Apollon Lykeios doit être plutôt considéré ici comme un dieu de la lumière. R.D. Dawe reconnaît que l'épithète Lykeios est souvent associée au loup, mais juge, lui aussi, l'association du

haut, selon laquelle Apollon Lykeios ne peut être qu'un « tueur de loups » : « Et toi, dieu qui détruis les loups, détruis l'armée de nos ennemis » (P. MAzoN, 19312 [1920] (n. 8), p. 115).

26 'L. Lupas, Z. Petre, Commentaire aux «Sept contre Thèbes» d'Escbyle, Bucarest, Paris, 1981, p. 54 .

27 Ce malheur qui frappe la cité est exprimé dans la lamentation funèbre chantée par le chœur, v. $822 \mathrm{~s}$.

28 JевB, 1902, loc. cit. (n. 22); KAMERBEeK, 1967, loc. cit. (n. 22). 
mot avec la lumière mieux en accord avec les autres images de cette partie de la parodos $^{29}$. On peut noter cependant que le mot $* \lambda \hat{v} \kappa \eta$, d'où dériverait

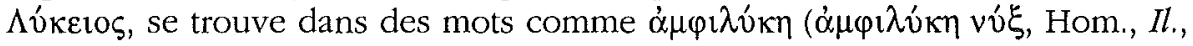
VII, 433), "épithète de la nuit déjà un peu lumineuse avant l'aurore », ou

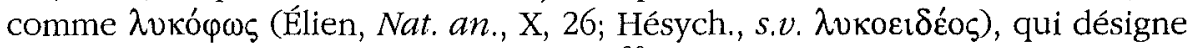
le «crépuscule du matin ou du soir ${ }^{30}$. Ces mots évoquent plutôt l'idée d'une faible lumière que d'une lumière vive, comme celle de l'éclair ou de la foudre de Zeus, des flambeaux d'Artémis ou de la torche de Bacchos. Mais, pour les Anciens, il existait un rapport entre une telle lumière et le loup, puisque, d'après Élien : «On appelle $\lambda$ voó $\omega_{\text {ş }}$ le moment de la nuit où seul le loup perçoit la lumière » (grâce à sa vue perçante); et il donne une définition semblable du mot $\alpha \mu \varphi \imath \lambda \hat{\kappa} \eta^{31}$. D'autre part, il est possible qu'il y ait, dans le texte de Sophocle, une relation entre la tournure $\Lambda v^{\prime} \kappa \varepsilon \iota^{\prime} \alpha{ }^{\prime} v \alpha \xi$ (v. 203) et l'adjectif $\Lambda$ v́к1 $\alpha$, «de Lycie » (v. 208), désignant le séjour d'Artémis ${ }^{32}$. On a donc peut-être ici une sorte de jeux de mots autour de l'épithète $\Lambda$ v́кદıs, sans qu'il soit possible de définir ce qu'il pouvait évoquer pour le poète et pour ses auditeurs.

Le sens que peut avoir l'épithète Lykeios dans l'Edipe Roi apparaîtra mieux si l'on examine le deuxième passage de la tragédie où Apollon est invoqué sous ce nom. En effet, alors qu'CEdipe vient de se rendre compte qu'il est peut-être le meurtrier de Laïos, Jocaste, le voyant angoissé et troublé, a décidé d'apporter des offrandes aux dieux de la cité. Devant le palais, elle adresse cette prière à Apollon (v. 918-923) : « Puis donc que mes conseils n'obtiennent rien de lui, c'est vers toi que je me tourne, ô dieu Lycien,

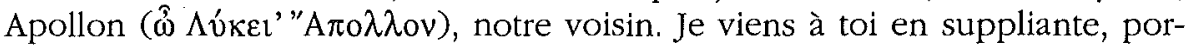
teuse de nos vœux. Fournis-nous un remède contre toute souillure. Nous nous inquiétons, à voir Edipe en désarroi, alors qu'il tient dans ses mains la barre de notre vaisseau ».

Le fait que Jocaste invoque Apollon devant le palais suppose la présence à cet endroit d'une effigie ou d'une autre représentation du dieu. On admet généralement qu'il s'agit du symbole d'Apollon Agyieus ${ }^{33}$. J.C. Kamerbeek estime que l'épithète Lykeios, dans cette prière de Jocaste, " n'est pas spécifique, mais se rapporte peut-être au caractère $\dot{\alpha} \lambda \varepsilon \xi \hat{i} \kappa \alpha \kappa \circ \zeta$ ou $\dot{\alpha} \pi$ o $\tau \rho$ ó $\pi \alpha$ to d'Apollon Agyieus " ${ }^{34}$. Il est vrai que Jocaste, en s'adressant à Apollon, dont

29 JEBB, 1902, loc. cit. (n. 22); R.D. DAwe, Sophocles Edipus Rex, Cambridge, 1982, p. 114.

30 P. Chantraine, Dictionnaire étymologique de la langue grecque, vol. 2, Paris, 1984, s. $v . * \lambda u ́ k \eta$.

31 ÉLIEN, Nat, an., X, 26.

32 JebB, 1902 (n. 22), p. 39; KAMERBEEK, 1967 (n. 22), p. 67.

33 Kamerbeek, 1967 (n. 22), p. 184; J.D. Denniston, D. Page, Aescbylus Agamemnon, Oxford, 1957, p. 167; Fraenkel, op. cit. (n. 22), p. 491.

34 Kamerbeek, loc. cit. (n. 33). 
le symbole était destiné à protéger le palais et ses habitants, espère l'aide et le secours du dieu. Mais elle ne sait pas, en prononçant cette prière, qu'Apollon lui-même, en exigeant par son oracle qu'on retrouve le meurtrier de Laïos, prépare la perte d'CEdipe et sa propre mort. Dans un premier temps, elle peut croire que sa prière a été exaucée : le messager de Corinthe vient annoncer la mort de Polybe, qu'elle prend pour une bonne nouvelle. Mais, peu après, viennent les révélations du serviteur de Laïos sur la naissance d'Edipe, qui entraînent la fuite de Jocaste dans le palais et son suicide. De même, Jocaste demande à Apollon Lykeios « un remède contre toute souillure »; le dieu va le lui accorder, mais pas dans le sens où elle le demande.

La prière de Jocaste est comme un signal annonçant que le moment de sa mort et de la chute d'CEdipe est proche. À partir de cette prière, les événements se précipitent: elle semble accélérer la marche des événements qui vont aboutir à la ruine des époux. Apollon Lykeios est, comme dans les Sept contre Thèbes, le dieu qui frappe cruellement celui qui invoque son secours, alors que celui-ci peut croire sa prière exaucée.

Dans l'Agamemnon d'Eschyle, on trouve une invocation à Apollon Lykeios, prononcée par Cassandre. Dans la partie parlée de la «scène de Cassandre » (v. 1178-1330), la jeune femme, après avoir évoqué le meurtre des enfants de Thyeste, mangés par leur père, puis annoncé la mort d'Agamemnon, se lamente sur son sort misérable et sur sa mort prochaine, dans un

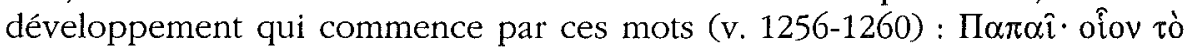

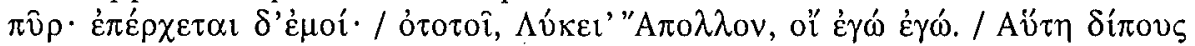

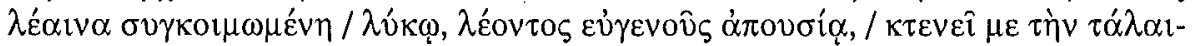
$v \alpha v$, «Ah! ah! quel est ce feu ? Et il marche sur moi... Apollon Lykeios, pitié, pitié pour moi ! - C'est elle, la lionne à deux pieds qui dormait avec le loup en l'absence du noble lion, c'est elle qui va me tuer, malheureuse ! »

On remarque d'abord que Cassandre, qu'Apollon a fait prophétesse (v. 1202), peut attendre la protection du dieu. Mais Apollon veut sa mort : «Et voici qu'aujourd'hui le prophète qui m'a fait prophétesse m'a lui-même conduite à ce destin de mort » (v. 1275-1276). C'est ce dieu qui va la perdre

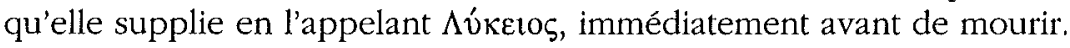

Deuxièmement, on note qu'après avoir invoqué Apollon Lykeios, Cassandre désigne Égisthe comme $\lambda \hat{\kappa} \kappa \varsigma_{\text {, }}$ « loup ». Certains commentateurs ${ }^{35}$ estiment qu'il y a un rapport entre l'épithète Lykeios d'Apollon et le fait qu'Égisthe soit appelé « loup ». Mais il reste à définir ce rapport et, d'abord, à comprendre pourquoi Égisthe est désigné ainsi. Il y a sans doute plusieurs explications possibles. Pour P. Vidal-Naquet, il est le loup, cruel et rusé qui, avec Clytemnestre, traque et tue Agamemnon à la façon d'un chasseur. Selon W.E. Higgins, il est le banni, le hors-la-loi perfide, qui gouverne à la manière

35 Cités par Fraenkel, op. cit. (n. 22), p. 581. 
du tyran que Platon compare à un loup, dans la République ${ }^{36}$. Mais le $\lambda \hat{v}$ xo Égisthe évoque aussi des loups de la mythologie.

On a déjà fait allusion à l'horrible festin préparé par Atrée pour son frère Thyeste, à qui il donna à manger la chair de ses enfants. Ce repas est mentionné à trois reprises dans la scène de Cassandre (v. 1090-1097, 1184-1193, 1217-1222), puis raconté par Égisthe lorsqu'il entre en scène (v. 1577-1611). Égisthe, lui-même fils de Thyeste ayant échappé au massacre, raconte comment, après ce funeste repas, Atrée les exila, son père et lui, loin d'Argos. Revenu d'exil, il a comploté la mort d'Agamemnon pour tirer vengeance du forfait commis par Atrée. On trouve dans la mythologie un repas comparable, celui du roi arcadien Lycaon, qui voulut faire manger à Zeus la chair d'un enfant et que Zeus, pour cela, au moins dans certaines versions du mythe, transforma en loup. Il existait un rituel apparenté, pratiqué dans le cadre du culte de Zeus Lykaios, sur le Mont Lycée, en Arcadie, au cours duquel un individu, qui avait, disait-on, mangé des viscères humains, se transformait en loup et vivait une certaine période loin de la société des hommes ${ }^{37}$. Égisthe fait penser aux loups des croyances arcadiennes : chassé d'Argós avec son père parce que celui-ci a consommé de la chair humaine, il est, après son exil, désigné comme loup. De plus, comme le roi Lycaon, Égisthe est un « loup » qui échoue. En effet, on se rappelle le «loup » Danaos, évoqué par Pausanias, qui, grâce au soutien d'Apollon Lykeios, écarte le roi Gélanor et prend le pouvoir à Argos. Le « loup » Égisthe, pour sa part, élimine Agamemnon et s'empare de son pouvoir (v. 1638-1639). La proximité, dans le texte d'Eschyle, du mot $\lambda u ́$ кos, désignant Égisthe (v. 1259), par rapport au nom d'Apollon Lykeios (v. 1257), pourrait indiquer que le dieu lui apporte son appui. C'est possible, car le fils de Thyeste, malgré toute l'antipathie qu'il peut inspirer, agit conformément à la justice, lorsqu'il tue Agamemnon pour venger l'horrible crime commis par Atrée ${ }^{38}$. Mais, on le verra dans l'Électre de Sophocle, le soutien d'Apollon Lykeios à Égisthe est provisoire : à cause du meurtre d'Agamemnon et de son union avec Clytemnestre, il sera lui-même tué par Oreste, placé à son tour sous la protection d'Apollon Lykeios.

36 P. Vidal-Naquet, Chasse et sacrifice dans l'« Orestie » d'Escbyle, in J.-P. Vernant, P. Vidal-Naquet, Mytbe et tragédie en Grèce ancienne I, Paris, 1972 [cité Vidal-Naquet, 1972a], p. 133-158, ici 146-147. W.E. Higgins, Wolf-god Apollo in the Oresteia, in PP, 31 (1976), p. 201-205, ici 202-203; Platon, Rép., 565d - 566a.

37 Sur le mythe de Lycaon et le rituel apparenté : Burkert, 1972 (n. 20), p. 98-108; Ph. Borgeaud, Recherches sur le dieu Pan, Genève, 1979, p. 41-51, 62-66; M. Jost, Sanctuatres et cultes d'Arcadie, Paris, 1985, p. 249-269; P. BONNECHERE, Le sacrifice bumain en Grèce ancienne, Athènes, Liège, 1994 (Kernos, Suppl. 3), p. 85-96. Sur le repas de Thyeste et ses rapports avec le mythe arcadien : Burkert, op. cit., p. 119-123. On évoquera plus loin le caractère « initiatique » du rituel du Mont Lycée : $c f . \$ 3$.

38 Denniston, Page, op. cit. (n. 33), p. 217 (ad Esch., Ag., 1607); Fraenkel, op. cit. (n. 22), p. 742 (ad EscH., $\mathrm{Ag} ., 1577$ ). 
L'Électre de Sophocle est la tragédie où le nom d'Apollon Lykeios est le plus souvent cité. Le dieu y est mentionné sous ce nom dans trois passages importants : au début du prologue (v. 7), vers le milieu de la pièce, dans une prière de Clytemnestre (v. 645, 655), et à la fin, dans une prière d'Électre (v. 1379).

L'épithète Lykeios apparaît d'abord dans le prologue, où le $\pi \alpha 1 \delta \alpha \gamma \omega \gamma o ́$, le " précepteur », arrivant à Mycènes avec Oreste et Pylade, présente à Oreste le

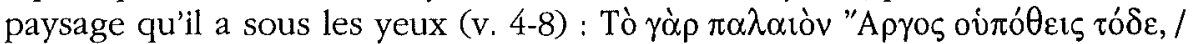

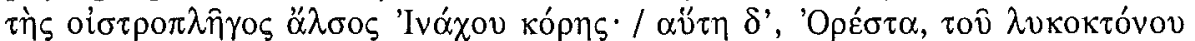

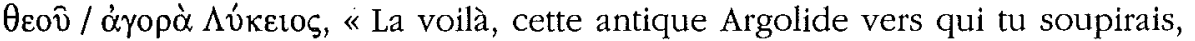
ce pourpris de la vierge, fille d'Inachos, taraudée par le taon. Voilà, Oreste, la Place Lycienne, vouée au dieu tueur de loups ».

On se souvient qu'il y avait à Argos un sanctuaire d'Apollon Lykeios, qui était le plus illustre et le plus ancien des monuments de la ville ${ }^{39}$. En se fondant en particulier sur ces vers d'Électre, on admet généralement que ce sanctuaire se trouvait sur l'agora d'Argos et que, pour cette raison, on appe-

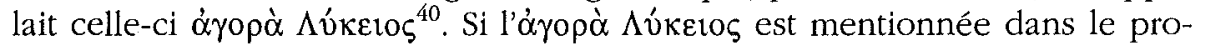
logue de la pièce, c'est probablement parce qu'Apollon, sous l'épithète de Lykeios, joue un certain rôle, voire un rôle important, dans l'action. De même, si le dieu est désigné ici comme $\lambda$ voко sens précis et directement en relation avec le drame. $\Lambda$ vкоктóvos, il n'y a pas de doute, signifie «tueur de loup(s) ». Mais de quel(s) loup(s) s'agit-il ? Selon une conception courante, on l'a vu, Apollon serait « tueur de loups » parce que, exerçant une fonction de dieu-berger, il tue les loups qui déciment les troupeaux. Mais, d'une part, le rôle de berger fait partie des attributions marginales d'Apollon ${ }^{41}$ et, d'autre part, il semble peu probable que, dans le

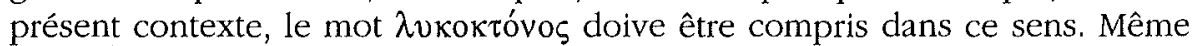
si l'on donne à l'expression "tueur de loups » le sens figuré de "destructeur d'ennemis », « protecteur contre les calamités », ce sens paraît trop vague et difficile à justifier ici ${ }^{42}$.

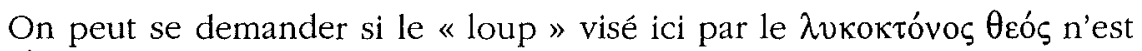
pas Égisthe, qui est appelé $\lambda$ úkos dans l'Agamemnon d'Eschyle, et qui va être tué à la fin de la pièce de Sophocle. Il est vrai que le dieu ne le tue pas luimême, il a ordonné à Oreste de le faire - celui-ci vient accomplir la ven-

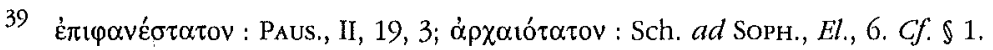

40 Wernicke, loc. cit. (n. 2), col. 59; Graf, op. cit. (n. 1), p. 222; D. Musti, M. Torelit, Pausania, Guida della Grecia, Libro 2, La Corinzia e l'Argolide, Fondazione Lorenzo Valla, 1986, p. 274.

41 W. Burkert, Apellai und Apollon, in $R h M, 118$ (1975), p. 1-21, ici p. 12, n. 45; Gershenson, op. cit. (n. 7), p. 10.

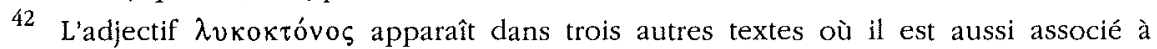
Apollon : Plut. Mor. 966a; Porph. Abst. I, 22; Anth. Pal. (Phaedim.) XIII, 22. Ces textes sont passablement plus récents que le prologue d'Électre; il est possible que l'adjectif y soit un emprunt au texte de Sophocle et ils ne nous aident pas à préciser le sens du mot. 
geance du meurtre de son père après avoir consulté l'oracle de Delphes. Apollon Lykeios est donc, dans cette tragédie, le dieu qui envoie Oreste tuer les assassins de son père. Sur la base de la définition des sens possibles de l'épithète Lykeios proposée plus haut, on peut considérer qu'Apollon est ici le « dieu-au-loup », le dieu qui envoie un loup pour tuer : ce «loup » est Oreste. Et le «loup » Oreste qui tue le «loup » Égisthe, cela signifie qu'Égisthe, qui a été protégé jusqu'à présent par Apollon Lykeios, est « lâché » maintenant par le dieu, qui accorde désormais son soutien à Oreste, car le moment est venu pour celui-ci de venger son père. Cela permet de comprendre les vers 6-7 sans donner à l'épithète $\Lambda$ v́kєı

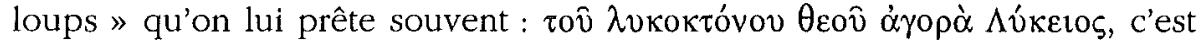
l'agora du " dieu-au-loup », celui qui envoie le loup tueur, mais qui peut aussi être « tueur de loups ». À l'appui de cette interprétation, on notera que, dans les Choéphores d'Eschyle, Électre, ce « double » d'Oreste ${ }^{43}$, dit que son cour

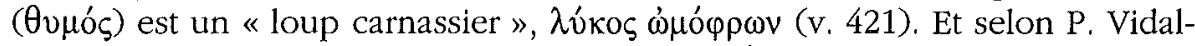
Naquet, Oreste, pour tuer le loup-chasseur Égisthe qui a causé la mort d'Agamemnon, recourt lui-même à la technique d'un chasseur ${ }^{44}$. On verra plus loin que, d'un autre point de vue aussi, Oreste peut être considéré comme un « loup » au service d'Apollon Lykeios.

En dehors du prologue, on trouve l'épithète Lykeios dans deux autres passages d'Électre : une prière de Clytemnestre (v. 634-659) et une prière d'Électre (v. 1376-1383). Clytemnestre, qui vit dans la peur du retour d'Oreste, a fait un rêve qui l'effraie ${ }^{45}$. Elle est sortie devant le palais, avec une esclave, pour apporter des offrandes à Apollon et lui adresser cette prière (v. 644656) : « Si les visions ambiguës que j'ai vues cette nuit en rêve ont un sens qui

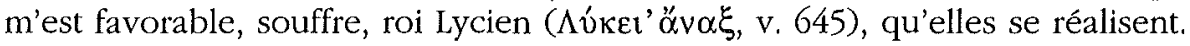
Mais si leur sens m'est hostile, retourne-les alors contre ceux qui me sont hostiles, et s'il se forme un complot pour m'expulser traîtreusement des richesses dont je jouis, ne le tolère pas, et, au contraire, fais que, vivante, je continue de posséder comme aujourd'hui, au cours d'une existence que rien ne vient troubler, le palais et le sceptre des descendants d'Atrée, tranquille, en compagnie des amis qui m'entourent et de tous ceux de mes enfants qui n'ont ni haine à mon endroit ni même chagrin trop amer. À ces voux,

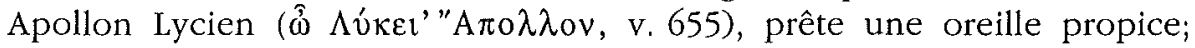
accorde-nous à tous le succès que nous implorons ». Clytemnestre parle de

43 VIDAL-NAQUET, 1972a (n. 36), p. 152. Certains philologues attribuent d'ailleurs ces mots à Oreste : R. Buxton, Wolves and Werewolves in Greek Thought, in J. Bremmer (éd.), Interpretations of Greek Mytbology, London, Sidney, 1987, p. 60-79, ici 61.

44 VIdAl-NaqueT, 1972a (n. 36), p. 146-147; p. 152.

45 Ce rêve est raconté par Chrysothémis à Électre (v. 417-423) : « On dit qu'elle aurait vu notre père, à nous deux, reparaître devant elle, et qu'il aurait planté dans notre foyer le sceptre qu'il portait jadis, avant qu'Égisthe le lui eût pris. De ce sceptre alors aurait jailli un laurier florissant, capable de couvrir à lui seul de son ombre toute la terre de Mycènes, » 
«visions ambiguës », car le rêve, suivant comment on l'interprète, peut être de bon ou de mauvais augure pour elle; il peut signifier, en particulier, ou bien qu'Égisthe, et sa descendance, continuera à régner sur Mycènes, ou bien, au contraire, qu'Oreste reprendra le pouvoir exercé par son père ${ }^{46}$. Clytemnestre, en invoquant Apollon Lykeios ${ }^{47}$, peut espérer que sa prière sera exaucée, car le dieu les a laissés, Égisthe et elle-même, vivre tranquilles de longues années, en profitant des richesses et du pouvoir qu'ils détiennent, malgré le meurtre d'Agamemnon et leur union honteuse. Mais, justement à cause de ce meurtre et de cette union, elle peut craindre que le rêve ne signifie le retour d'Oreste, venu pour châtier les assassins de son père.

Comme Jocaste dans CEdipe Ro $i^{48^{\prime \prime}}$, elle peut croire d'abord que sa prière a été entendue : le «précepteur » vient lui annoncer qu'Oreste est mort. Mais le soulagement de Clytemnestre est de courte durée. Peu après, Oreste arrive, se fait reconnaître d'Électre, puis entre dans le palais avec ses compagnons, pour accomplir sa vengeance. À ce moment, Électre, restée à la porte du palais, prononce la prière suivante (v. 1376-1383), qui est une sorte d'« écho » à la prière de Clytemnestre : « Seigneur Apollon, prête-leur une oreille favorable - et à moi aussi, qui me suis bien souvent présentée devant toi, portant dans mes mains suppliantes le peu que je pouvais t'offrir. Cette fois, Apollon Lycien ( $\hat{\hat{\omega}} \Lambda \hat{v} \kappa \varepsilon l$ ' "A $\mathrm{\pi} \sigma \lambda \lambda o v$ ), sans autres offrandes, je te prie, te supplie et t'implore de prêter à nos desseins ton plus généreux concours et de faire voir aux mortels quels châtiments les dieux réservent à l'impiété ». Immédiatement après cette prière, Clytemnestre, puis Égisthe, sont tués par Oreste. Ici aussi, comme dans la prière de Jocaste, l'invocation à Apollon Lykeios est le signal que leur mort est imminente.

Comme on l'a vu à plusieurs reprises, Apollon Lykeios est généralement considéré comme un dieu protecteur contre les ennemis et les malheurs de toutes sortes. Dans les passages de tragédies qui ont été examinés jusqu'ici, il révèle un caractère ambigu. Il est effectivement invoqué comme un dieu protecteur, mais, à la suite d'une prière qui lui est adressée, il provoque la mort brutale ou un profond malheur pour celui qui l'implore. Dans un premier temps, un événement fait croire au suppliant qu'il a été entendu favorablement par le dieu, mais, peu après, la mort ou le malheur le frappe brutalement. Certes, le dieu semble protéger Égisthe et Clytemnestre pendant un certain temps, en les laissant assassiner Agamemnon et jouir du pouvoir et des richesses de celui-ci, mais il envoie ensuite Oreste les tuer ${ }^{49}$. On le verra

\footnotetext{
46 Kamerbeek, 1974 (n. 22), p. 93; J.H. Kells, Sopbocles Electra, Cambridge, 1973 , p. 134 .

47 Cf. supra, n. 33.

48 Les prières de Jocaste et de Clytemnestre sont comparées par Kamerbeek, 1967 (n. 22), p. 182; 1974 (n. 22), p. 92.

49 Cet aspect d'Apollon, qui tue celui qui vient, en suppliant, lui offrir un sacrifice, a été mis en évidence, à propos du meurtre de Néoptolème dans le sanctuaire de Delphes, par Burkert, 1972 (n. 20), p. 136-138; M. Detienne, J. Svenbro, Les loups au festin ou la
} 
par la suite, le loup, pour les Grecs, est perfide. Apollon Lykeios agit avec la perfidie du loup, lorsque celui qui l'implore, croyant que sa prière est exaucée, est détrompé cruellement, ou lorsque le $\lambda \hat{\text { ko }}$ qu'il a protégé est soudain la victime du $\lambda$ vкоктóvos $\theta$ eós.

Une seule personne est épargnée après l'avoir prié : c'est Électre. Mais Électre, dans sa prière, demande le succès d'Oreste. Or Apollon Lykeios, comme on va le voir, est le dieu qui guide Oreste et lui prescrit comment accomplir sa vengeance. Là encore, le rôle qu'il joue auprès du jeune homme se caractérise, d'une autre façon, par son ambiguité.

\section{Apollon Lykeios, « dieu de l'initiation »}

Étudiant l'Orestie d'Eschyle, P. Vidal-Naquet a montré qu'Oreste, dans les Choépbores, se caractérise comme un éphèbe, « apprenti-homme et apprenti-guerrier usant de la ruse avant d'acquérir la morale de la bataille »; l'éphébie ne doit alors pas être comprise comme une institution à caractère militaire et civique, telle qu'elle existait en particulier à Athènes, mais comme la « trace », au niveau du mythe, d'un rituel initiatique marquant le passage d'un jeune homme de l'enfance à l'âge adulte ${ }^{50}$. De tels rites d'initiation, par lesquels un individu accède à la communauté des adultes, sont bien connus dans les sociétés dites «primitives », et A. Van Gennep a établi qu'ils s'articulent en trois phases ${ }^{51}$ : phase de « séparation », où les jeunes gens en âge d'être initiés sont soustraits au monde de l'enfance; phase de « marge », que les « initiants » passent à l'écart du groupe social et qui se caractérise par une inversion des valeurs de la communauté; phase d' « agrégation », au cours de laquelle les jeunes gens sont intégrés à la communauté adulte, dont ils deviennent membres à part entièrẹ.

En outre, à partir d'une étude de la cryptie spartiate - institution dans le cadre de laquelle certains jeunes Spartiates, au moment de la puberté, vivaient isolés dans la nature sauvage et s'adonnaient au meurtre des hilotes - P. Vidal-Naquet a montré que l'éphèbe, tel qu'il apparaît dans les mythes, est, comme le crypte, un « anti-hoplite » : sans arme ou légèrement armé, il vit

Cité impossible, in M. Detienne, J.-P. Vernant, La cuisine du sacrifice en pays grec, Paris, 1979, p. 215-237, ici 235-236; M. DETIENNE, L'Apollon meurtrier et les crimes de sang, in QUCC, 51 (1986), p. 7-17, ici 10 sq.. Le manuscrit du présent article était achevé au moment de la parution de l'ouvrage de M. Detienne, Apollon le conteau à la main. Une approche expérimentale du polytbéisme grec, Paris, 1998.

50 P. Vidal-Naquet, 1972a (n. 36), p. 151; ID., Le chasseur noir et l'origine de l'éphébie atbénienne, in Le chasseur noir, Paris, $1983^{2}$ [1981] [cité VIDAL-NAQUET, 1983a], p. 151-175, ici 151-154; ID., Le cru, l'enfant grec et le cuit, ibid. [cité VIDAL-NAQUET, 1983b], p. 177-207, ici 193-196.

51 A. VAN Gennep, Les rites de passage, Paris, 1909. Les caractéristiques des trois phases de l'initiation sont résumées par C. Calame, Les cbœurs de jeunes filles en Grèce archaique. I. Morphologie, fonction religieuse et sociale, Rome, 1977, p. 32-38. 
isolé ou en petits groupes; en marge de la cité, il fréquente la montagne ou les zones frontières; il pratique le meurtre ou le combat singulier, et se sert de la ruse pour dominer ses adversaires. Il appartient au domaine du désordre et du « sauvage $»^{52}$.

D'autre part, comme l'a indiqué W. Burkert (à propos de la fête dorienne des Apellai $^{53}$, il existait un rapport entre Apollon et l'éphébie, et l'on peut voir en Apollon l'« archétype » de l'éphèbe prenant part à une cérémonie d'initiation. H.S. Versnel ${ }^{54}$, pour sa part, considère Apollon comme l'image des éphèbes pendant la période où ils vivent retirés dans le monde sauvage, en marge de la cité; le dieu revient chaque année d'une région lointaine, et ce retour peut être interprété comme l'image du retour des jeunes gens à la fin de leur période de marge, lorsqu'ils sont intégrés dans la société des adultes ${ }^{55}$.

Dans les Choéphores et les Euménides d'Eschyle, A. Bierl a, lui aussi, mis en évidence des traits qui caractérisent Oreste comme un éphèbe en période d'initiation et il a étudié le rôle d'Apollon dans l'initiation d'Oreste ${ }^{56}$. Des traits comparables se manifestent dans l'Électre de Sophocle; ils apparaissent tous dès le prologue et sont repris dans la suite de la pièce.

Dans les Cboépbores et les Euménides, Oreste est présenté, selon A. Bierl, comme «un adolescent au seuil de l'âge adulte » et cela est indiqué en particulier par le fait qu'il est désigné aussi bien comme $\pi \alpha \hat{\imath} \varsigma$ ou $\tau \dot{\varepsilon} \kappa v o v$, que comme $\alpha \dot{\alpha} \rho^{57}$. C'est aussi le cas dans l'Électre de Sophocle, mais ces trois termes apparaissent dans des conditions particulières sur lesquelles on reviendra plus loin. De plus, dans la pièce de Sophocle, Oreste est caractérisé précisément comme un éphèbe, il est celui qui est parvenu à l'n̋ $\beta \eta$ :

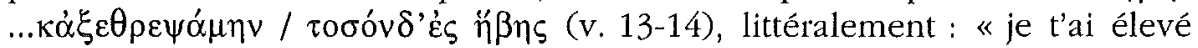
jusqu'à ce point de l'adolescence où tu es », lui dit le précepteur dans le

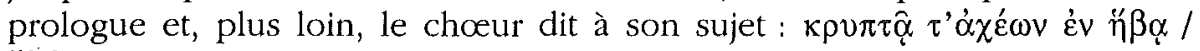
ő $\lambda \beta ı$ s, « celui dont la jeunesse heureuse demeure à l'abri des chagrins » (v. 159-160). Un autre élément caractérise Oreste comme un très jeune

52 Vidal-Naquet, $1983 a$ (n. 50), p. 159-164; p. 173-174; 1983b (n. 50), p. 201-202. P. Vidal-Naquet a également mis en évidence des traits «éphébiques », comme le recours à la ruse ou la fréquentation du monde sauvage, dans le personnage de Néoptolème, tel qu'il apparaît dans le Philoctète de Sophocle : P. Vidal-NaqueT, 1972b : Le "Philoctète" de Sopbocle et l'épbébie, in J.-P. VeRnaNT, P. VIDAL-NAqueT, Mythe et tragédie en Grèce ancienne I, Paris, 1972, p. 159-184.

53 BURKERT, 1975 (n. 41), p. 10-11, 17-18, 19-20.

54 H.S. VERSNel, Apollo and Mars One Hundred Years after Roscber, in Inconsistencies in Greek and Roman Religion II. Transition and Reversal in Myth and Ritual, Leiden, 1993, p. 315-316.

55 On mentionnera plus loin les travaux de F. Graf et de M. Jameson sur les liens d'Ápollon Lykeios avec l'éphébie.

56 A. BIERL, Apollo in Greek Tragedy: Orestes and the God of Initiation, in J. Solomon (éd.), Apollo, Origins and Influences, Tucson, London, 1994, p. 81-96, ici 85 sq.

57 BierL, loc. cit. (n. 56), p. 85. 
homme : c'est le fait qu'il soit accompagné, ou plutôt guidé, par le $\pi \alpha \downarrow \delta \alpha \gamma \omega \gamma o ́ \varsigma$, le « précepteur», littéralement « celui qui conduit l'enfant ». La dépendance du jeune homme à l'égard de ce personnage est manifeste : c'est lui qui a emmené Oreste loin du palais après le meurtre d'Agamemnon, c'est lui maintenant qui le ramène en Argolide afin qu'il venge son père (v. 1-13). Oreste lui expose le plan qui va lui permettre d'accomplir sa vengeance et lui demande de le corriger s'il fait une erreur (v. 30-31); plus loin, il accepte ses remontrances (v. 1326-1337). Enfin, c'est le précepteur qui incite Oreste et Pylade à passer à l'action (v. 21-22; 1337-1338; 1367-1368). On peut noter également que, dans la pièce de Sophocle comme dans les Choépbores, Oreste offre sur la tombe de son père une boucle de ses cheveux (v. 52, 901). Certes, il s'agit d'une offrande de deuil, mais elle évoque également un rite d'adolescence ${ }^{58}$.

Dans les premiers vers du prologue, Oreste est présenté comme un exilé qui revient dans son pays et peut « voir enfin ces lieux qui toujours ont fait son envie » (v. 2-3); le précepteur rappelle comment, l'ayant reçu des mains d'Électre, il l'a emmené après le meurtre de son père (v. 11-13). D'autre part, Oreste (de même que ses compagnons, le précepteur et Pylade) se fait passer pour un étranger ( $\xi \varepsilon \dot{\varepsilon} \vee \zeta_{\text {) }}$, afin de ne pas être reconnu par les occupants du palais (p. ex. : v. 44, 1112, 1180, 1206, 1442). Or ces deux traits d'Oreste, qui se trouvent aussi dans les Choéphores ${ }^{59}$, évoquent un « initiant » pendant sa période de «marge » : enlevé, enfant, au foyer de ses parents, il est resté longtemps dans une région éloignée de sa cité.

Comme on l'a vu précédemment, P. Vidal-Naquet a montré que la ruse est un des traits typiques du comportement de l'éphèbe; il a mis en évidence que, dans les Choéphores, Oreste, par les aspects rusés de son combat, se caractérise comme un éphèbe ${ }^{60}$. Dans l'Électre de Sophocle, le comportement rusé d'Oreste est également manifeste. Dans le prologue, Oreste révèle au précepteur le plan qu'il a mis au point pour châtier les assassins de son père. Évoquant l'oracle de Delphes, auquel il s'est adressé pour savoir com-

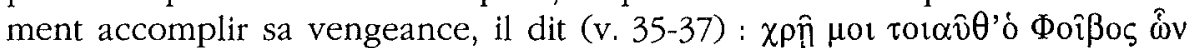

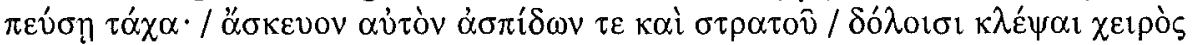

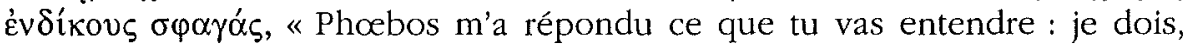
seul, sans bouclier, sans armée, par la ruse, en dissimulant, pourvoir au juste sacrifice qui est réservé à mon bras ".

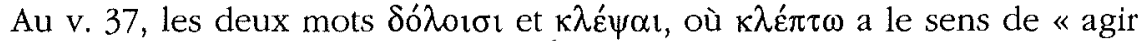
avec dissimulation ou avec perfidie ${ }^{61}$, insistent sur le comportement rusé

58 Esch,, Choéph., 6-7; Bierl, loc. cit. (n. 56), p. 85; Vidal-Naquet, 1972a (n. 36), p. 151152; $1983 \mathrm{a}$ (n. 50), p. 155.

59 Biert, loc. cit. (n. 56), p. 86, 90.

60 Vidal-Naquet, 1983a (n. 50), p. 159-163; 1972a (n. 36), p. 151-153. Brerl, loc. cit. (n. 56), p. 89-90.

61 H.G. Liddell, R. Scott, A Greek-English Lexicon, Oxford, $1940^{9}$, s.v. к $\lambda \varepsilon ́ \pi \tau \omega$, IV. 
que le dieu exige d'Oreste. Un peu plus loin dans le prologue, le verbe $\kappa \lambda \varepsilon ́ \pi \tau \omega$ est à nouveau utilisé dans le même sens. Oreste annonce qu'il apportera au palais la nouvelle de sa mort : « Je reviendrai ensuite (...) pour leur apporter

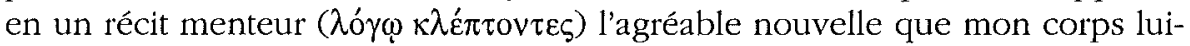
même n'est plus » (v. 53-57). Enfin, à la fin de la pièce, le jeune homme est

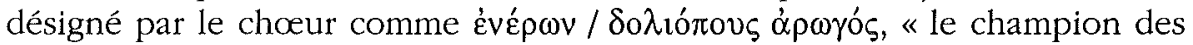
morts à la marche perfide » (v. 1391-1392).

Un autre mot du v. 37 mérite qu'on s'y arrête : $\sigma \varphi \alpha \gamma \alpha ́$ s, que P. Mazon traduit par " sacrifice », mais qui signifie en premier lieu « égorgement " ${ }^{62}$. Or, selon Aristote, le loup, pour tuer sa proie, l'égorge, $\sigma \varphi \alpha \zeta_{\varepsilon} \varepsilon^{63}$. Dolon, l'espion troyen revêtu d'une peau de loup, qui s'apprête à couper des têtes dans le

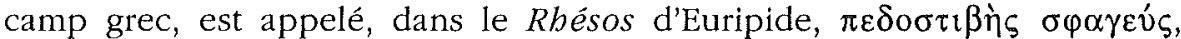
l'«égorgeur qui avance en rampant " ${ }^{64}$. Toujours selon Aristote, le loup est

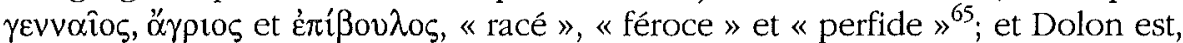
d'après son nom, « le Rusé " ${ }^{66}$. Le loup est donc un égorgeur perfide, et ce qu'Apollon demande à Oreste, c'est justement d'égorger ses victimes avec perfidie : cela semble confirmer l'interprétation proposée plus haut, selon laquelle Oreste est, dans l'Électre de Sophocle, le "loup » envoyé par Apollon Lykeios pour tuer les assassins de son père. À propos de la cryptie spartiate, H. Jeanmaire écrit : «Ce à quoi est obligé le cryptos (...), c'est à mener quelque temps (...) l'existence du loup. Le comportement qui lui est

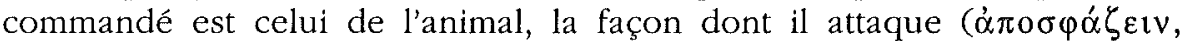
óvoıpeiv), celle du fauve ${ }^{67}$. Ainsi, Apollon, lorsqu'il demande à Oreste d'exécuter ses victimes à la manière perfide du loup, lui prescrit d'agir avec la sauvagerie propre à un « initiant » dans sa période de marge. Cependant, il ne

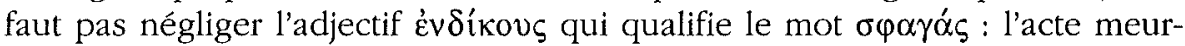
trier qu'Oreste va accomplir sur l'ordre du dieu est désigné comme « conforme à la justice, légitime ${ }^{68}$. C'est un point sur lequel on reviendra. À propos des traits éphébiques d'Oreste, il faut encore noter qu'au v. 36, Apollon prescrit au jeune homme d'accomplir sa vengeance «seul, sans bouclier, sans armée ». Or, le combat solitaire et sans l'armement lourd de

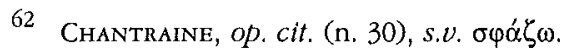

63 Aristt, Hist. an., IX, 6, 612b; cf. Detienne, Svenbro, loc. cit. (n. 49), p. 223-224.

64 Eur., Rbésos, 254, cité par Detrenne, Svenbro, loc. cit. (n. 49), p. 230-231; cf. L. Gernet, Dolon le loup, in Mélanges F. Cumont, Bruxelles, 1936, repris dans Anthropologie de la Grèce antique, Paris, 1982, p. 201-223, ici 209-211.
}

65 Aristr., Hist. an., I, 1, 488 b; traduction : P. Louis, Aristote, Histoire des animaux, tome 1, Paris, 1964, p. 8.

66 Gernet, loc. cit. (n. 64); Detienne, Svennbro, loc. cit. (n. 49), p. 230.

67 H. Jeanmaire, Couroi et Courètes. Essai sur l'éducation spartiate et les rites d'adolescence dans l'Antiquité bellénique, Lille, Paris, 1939, p. 554.

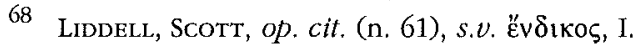


l'hoplite est aussi, selon P. Vidal-Naquet, un des traits caractéristiques de l'éphèbe ${ }^{69}$.

La ruse d'Oreste et de ses compagnons consiste d'abord à se faire passer pour des étrangers, afin de ne pas être reconnus, puis de faire croire les occupants du palais à la mort d'Oreste. Ce thème est présenté dès le prologue : le précepteur, se faisant passer pour un Phocidien, entrera dans le palais et racontera qu'Oreste est mort ( $v, 38-50)$; celui-ci arrivera ensuite, apportant une urne censée contenir les cendres de son propre corps (v. 53-58). Après être ainsi «mort en paroles » ( $\lambda{ }^{\prime} \gamma \omega \theta \alpha v \omega ́ v$, v. 59), il réapparaîtra «quelque jour bien vivant (...) à la vue de ses ennemis » (v. 65-66). Cette fausse mort d'Oreste fera ensuite l'objet d'un long récit du précepteur à Clytemnestre (v. 660-763). Puis, Oreste annoncera à Électre qu'il apporte les cendres du corps de son frère; vivement ému par le désespoir de la jeune femme, il ne pourra s'empêcher de se faire reconnaître par elle (v. 1098-1231). Comme l'a noté A. Bierl à propos des Choépbores, où Oreste recourt à une ruse semblable, la mort suivie de renaissance est un des motifs typiques des rites d'initiation. La phase de marge, comme l'indique C. Calame, " est en général comprise comme une période de mort, une mort initiatique (..), toujours liée à une renaissance ». Celle-ci, selon H.S. Versnel, correspond à la fin de la période de marge, au moment où les initiés reviennent des régions éloignées où ils se trouvaient et sont intégrés à la communauté des adultes ${ }^{70}$.

On peut considérer que la « résurrection » d'Oreste se produit au

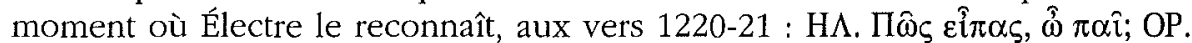

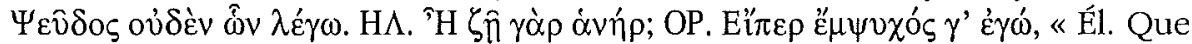
dis-tu, mon enfant? Or. Mais rien qui ne soit vrai. Él. Alors Oreste (littéralt l'homme) vit? Or. Oui, puisque je respire ». Or, à une exception près (v. 1430), Oreste n'est appelé $\pi \alpha \hat{\imath} \varsigma$ ou đékvov que jusqu'au v. 1220 ( $\pi \alpha \hat{\imath} \varsigma$ :

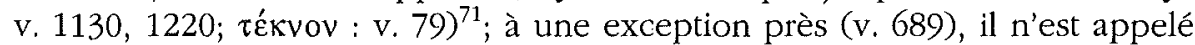
ávíp que depuis le v. 1221 (v. 1221, 1342, 1398, 1461, 1476). Avant le v. 1221, il est appelé $\alpha v \eta ́ \rho$ lorsqu'il est question d'exploits qu'il a réalisés; dans le récit de la «mort » d'Oreste, le précepteur dit à Clytemnestre (v. 688-689) : « je ne sais vraiment comment je te pourrais rapporter seulement quelques-uns (...)

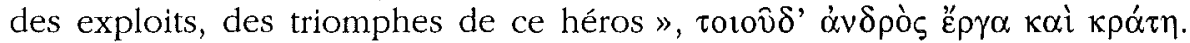
Ainsi, la fausse mort d'Oreste semble l'avoir fait passer de l'état d'enfant à celui d'homme ${ }^{72}$. Mais cela n'est qu'une première étape de son « agrégation » à la communauté des adultes. En effet, pour conquérir sa place à part entière

69 VIDAL-NAQUET, $1983 a$ (n. 50), p. 161-162, 173-174.

70 Bierl, loc. cit. (n. 56), p. 90; Calame, op. cit. (n. 51), p. 37; Versnel, loc. cit. (n. 54); voir aussi : BONNECHERE, op. cit. (n. 37), p. 21-26.

71 On n'a retenu ici que les cas où ces mots signifient « enfant » et non « fils ».

72 Le même changement de terminologie, indiquant le passage de Néoptolème du statut d'enfant à celui d'adulte dans le Pbiloctète de Sophocle, et montrant que le jeune homme « a traversé au cours de la pièce l'initiation éphébique », a été relevé par VIDALNAquet, 1972b (n. 52), p. 172. 
dans la société, c'est-à-dire retrouver le trône de son père, il doit encore accomplir l'acte, voire l'exploit, que l'on attend de lui, tuer les assassins de son père.

F.I. Zeitlin, évoquant les aspects éphébiques d'Oreste tels qu'ils apparaissent dans les Choéphores et les Euménides d'Eschyle, estime que le crime d'Oreste, le meurtre de sa mère, est l'acte que le jeune homme doit accomplir pour se détacher du monde de son enfance; sa période de marge commence après ce crime et «se termine lorsque, réincorporé dans la société au troisième stade du rite de passage, il retourne à Argos (...) pour succéder à son père ${ }^{73}$. Si l'on examine l'Électre de Sophocle, il semble préférable de situer le début de la période de marge d'Oreste au moment où celui-ci, enfant, a été emmené loin du palais de son père, c'est-à-dire loin du monde de son enfance. Adolescent, il revient. Certes, ce retour ne correspond pas au moment de son «agrégation » à la société. En effet, le foyer où il rentre est, comme le dit F.I. Zeitlin à propos des Cboéphores, « un espace rendu sauvage par l'action de sa mère ${ }^{74}$ et, pourrait-on ajouter, par l'action du $\lambda$ úko Égisthe; dans cet espace marqué par la sauvagerie ${ }^{75}$, Oreste va commettre «l'acte sauvage entre tous ${ }^{76}$, le meurtre de sa mère. Dans l'Électre de Sophocle, le crime commis par Oreste a un caractère ambigu. Il est un acte sauvage, accompli avec les ruses et le comportement du loup caractéristiques de l'éphèbe, mais, on l'a vu, il est aussi qualifié d'ěv $\delta ı \kappa_{\text {o }}$ et apparaît par conséquent comme l'exploit qu'Oreste, devenu homme, doit réaliser pour conquérir sa place légitime sur le trône d'Argos et obtenir ainsi son intégration complète dans la société. Donc, dans la pièce de Sophocle, on peut considérer que la vengeance d'Oreste marque la fin de son initiation.

Si l'on accepte cette interprétation, le comportement de loup prescrit à Oreste par Apollon Lykeios peut être mis en parallèle avec la transformation en loup qui se produisait dans le cadre du culte arcadien de Zeus Lykaios, et dont W. Burkert a montré le caractère initiatique ${ }^{77}$ : un jeune homme, transformé en loup, passait un certain temps dans le monde sauvage; à son retour, il était intégré dans la société des adultes.

73 F.I. ZEITLIN, The Dynamics of Misogyny: Mytb and Mythmaking in the Oresteia, in Aretbusa, 11 (1978), p. 149-184, ici 161.

74 Zeitlin, loc. cit. (n. 73).

75 Il' n'est d'ailleurs pas essentiel, du point de vue de l'initiation éphébique, que le palais où arrive Oreste soit un lieu "sauvage »; en effet, comme l'a montré C. Calame, Prairies intoucbées et jardins d'Apbrodite: espaces initiatiques en Grèce, in L'initiation, Actes du Colloque international de Montpellier, 11-14 avril 1991, tome 2, Montpellier, 1992, p. 103-118, ici 104-105, les espaces où se déroule le passage de l'enfance à l'état adulte n'appartiennent pas nécessairement au domaine sauvage.

76 ZeitulN, loc. cit. (n. 73).

77 Burkert, 1972 (n. 20), p. 104-105; Borgeaud, op. cit. (n. 37), p. 62-65; BonNechere, op. cit. (n. 37), p. 89-96; voir aussi Gernet, loc. cit. (n. 64), p. 204-206. 
Il reste encore à examiner le rôle d'Apollon dans l'initiation d'Oreste. On se souvient que, dès le début du prologue, est évoquée « la Place Lycienne

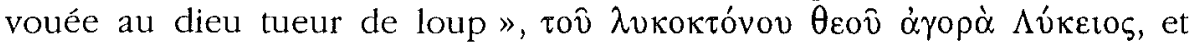
qu'Apollon Lykeios peut être considéré comme le dieu qui envoie le «loup » Oreste tuer le «loup " Égisthe. On a vu qu'Apollon, par son oracle de Delphes, a prescrit au jeune homme un comportement qui évoque celui d'un crypte spartiate ou d'un éphèbe. Oreste a passé son exil en Phocide : la période de marge du jeune homme est donc associée au domaine de l'Apollon delphique. Le dieu intervient également dans la mort fictive d'Oreste : elle s'est produite au cours des Jeux Pythiques (v. 49; 682); c'est Apollon qui en est responsable : « quand un dieu veut du mal à un homme, celui-ci (...) ne peut lui échapper» (v. 696-697). Enfin, Oreste, devenu homme après sa fausse mort, exécute la vengeance vers laquelle le dieu l'a conduit; cette vengeance est accomplie après une prière à Apollon Lykeios, où Électre demande au dieu son concours ${ }^{78}$. À travers toutes ces interventions, Apollon Lykeios se présente clairement comme le dieu qui guide l'éphèbe Oreste pendant son initiation.

Dans les Choépbores et les Euménides, selon A. Bierl, Apollon est le « dieu de l'initiation », qui guide l'éphèbe Oreste, mais il manifeste à l'égard de son protégé un comportement « ambivalent et négatif ». En effet, «malgré son aide et ses directives, Apollon (...) n'est pas capable de mener l'initiation d'Oreste à son terme "; la phase d'intégration du jeune homme à la société est confiée à Athéna ${ }^{79}$. Dans la pièce de Sophocle, si l'on considère la vengeance d'Oreste comme l'exploit qui lui permet de gagner sa place d'adulte dans la société, on doit admettre qu'Apollon Lykeios le conduit jusqu'à la fin de son initiation. Il subsiste cependant un doute quant au rôle joué par le dieu auprès du jeune homme.

Apollon Lykeios, on l'a vu, peut être aussi bien le dieu « au loup » que le dieu « tueur de loups ». En conséquence, à la fin de la pièce, on peut se demander si Oreste est vraiment parvenu avec succès au terme de son initiation, sous la protection d'Apollon Lykeios ou si, après avoir, sur l'ordre du dieu, tué ses ennemis à la manière d'un loup, il n'est pas lui-même menacé par le $\lambda$ ขкоктóvos $\theta \varepsilon o ́ s$. Ce doute au sujet du destin d'Oreste apparaît également dans les différentes interprétations qui ont été faites des derniers vers de la tragédie de Sophocle, qui sont prononcés par le coryphée (v. 1508-

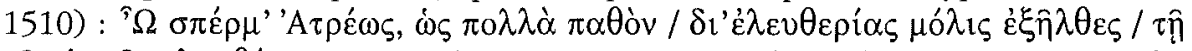

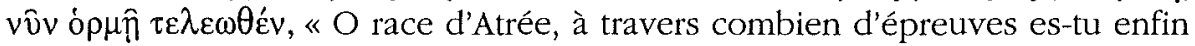
à grand-peine parvenue à la liberté ! L'effort de ce jour couronne ton histoire ». Les commentateurs modernes de la pièce s'accordent, pour le sens du passage, avec la traduction de P. Mazon: Oreste, le représentant actuel de la race d'Atrée, après avoir éliminé l'usurpateur Égisthe, est prêt à occuper sa

\footnotetext{
78 Cf. 2.

79 Bierl, loc. cit. (n. 56), p. 85, 87, 92.
} 
place légitime sur le trône d'Argos. Mais il y a plusieurs interprétations possibles du mot $\tau \varepsilon \lambda \varepsilon \omega \theta \varepsilon \dot{v} v$ : pour J.C. Kamerbeek, il signifie ici « confirmé <dans la possession de tes droits royaux > »; pour R.C. Jebb, il veut dire « accompli », « rendu parfait » et, selon lui, « le mot est appliqué à ceux qui ont atteint la maturité du corps et de l'esprit ${ }^{80}$. Le même auteur ne voit dans les derniers vers de la pièce « aucun signe de troubles à venir » et les oppose à la fin des Choéphores: «Où donc s'achèvera, où s'arrêtera, enfin endormi, le courroux d'Até ? ${ }^{81}$. J.H. Kells, cependant, trouve qu' « il est difficile de ne pas déceler de l'ironie » dans les vers de Sophocle, et évoque à ce propos le sinistre jeu

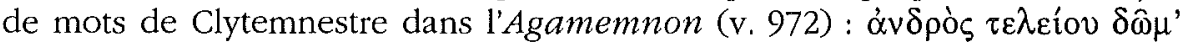

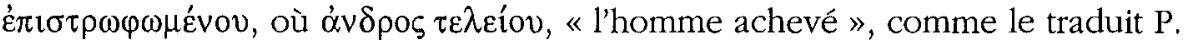
Mazon, désigne Agamemnon sur le point d'être tué ${ }^{82}$.

Cette incertitude relative au sort d'Oreste pourrait refléter le caractère double de son crime. Il tue Égisthe, l'assassin et l'usurpateur du trône de son père, mais il tue aussi sa mère : la gravité de ce matricide est mise en lumière dans les deux autres tragédies qui parlent des conséquences de la vengeance d'Oreste, les Euménides d'Eschyle et l'Électre d'Euripide, où Oreste est poursuivi par les Érinyes non pas pour le meurtre d'Égisthe, mais pour celui de sa mère. Cependant, si l'on considère le texte de Sophocle, et non les interprétations que l'on peut en faire, il semble que le poète ait voulu mettre l'accent, dans cette tragédie, non pas sur les aspects problématiques de la vengeance d'Oreste, mais sur son caractère légitime et sur le droit du jeune homme à occuper le trône d'Argos ${ }^{83}$. Oreste serait alors réellement, à la fin de la pièce, $\tau \varepsilon \lambda \varepsilon \omega \theta \varepsilon i$ s, homme « accompli » ou, selon notre interprétation, parvenu avec succès au terme de son initiation.

Cependant, l'action d'Apollon Lykeios auprès d'Oreste se caractérise

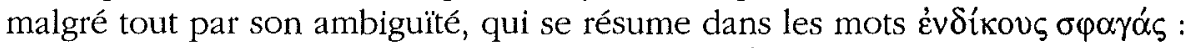

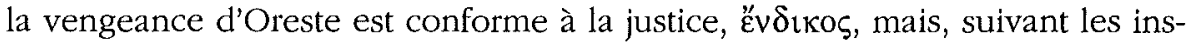
tructions du dieu, le jeune homme l'accomplit en adoptant le comportement rusé et la manière de tuer, $\sigma \varphi \alpha ́ \zeta \varepsilon ı v$, du loup. L'ordre légitime rétabli à Argos grâce à l'intervention d'Apollon Lykeios s'oppose à la sauvagerie du comportement dicté à Oreste par le dieu.

Il reste à examiner une tragédie où est invoqué Apollon Lykeios : les Suppliantes d'Eschyle. Danaos et ses filles, fuyant l'Égypte et les fils d'Égyptos, qui veulent les épouser de force, ont abordé à Argos et ont supplié le roi du pays de leur accorder l'asile. Alors que les Argiens viennent d'accéder à leur requête, le chour des Danaïdes, en remerciement de cette décision, appelle de ses vœux des bienfaits pour Argos. Dans cette invocation, on peut

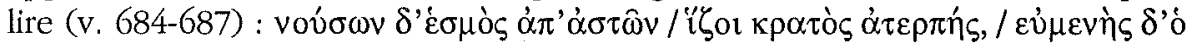

\footnotetext{
80 KaMerbeek, 1974 (n. 22), p. 194; JebB, 1907 (n. 22), p. 203.

81 JевB, loc. cit. (n. 80); Esch., Choéph., 1074-1076.

82 KELls, op. cit. (n. 46), p. 231.

83 JebB, 1907 (n. 22), p. XL-XL; KAMERBEEK, 1974 (n. 22), p. 17.
} 


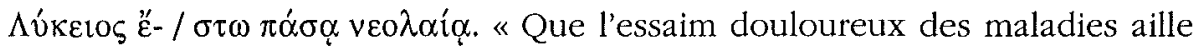
se poser loin du front des Argiens; et qu'Apollon Lycien soit propice à tous leurs enfants!». Immédiatement après que les Danaïdes ont prononcé ces mots, Danaos voit les vaisseaux des fils d'Égyptos approcher des côtes de l'Argolide. Il assure ses filles, épouvantées par cette nouvelle, que les Argiens les défendront. Les Danaïdes ayant traité les fils d'Égyptos de « chiens »

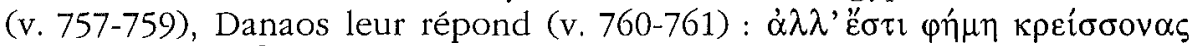

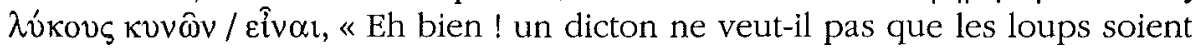
vainqueurs des chiens? ». On peut considérer que les "loups» sont ici les $\operatorname{Argiens}^{84}$ qui, en accordant droit de cité aux Danaïdes, se sont engagés à les défendre contre leurs ennemis. Mais on peut aussi admettre que ce terme désigne Danaos et les Danaïdes, qui sont les ennemis héréditaires d'Égyptos et de ses fils; d'ailleurs, les jeunes filles se réclament au début de la pièce de leur origine argienne (v, 274-323). D'autre part, Apollodore raconte comment les fils d'Égyptos arrivèrent à Argos et demandèrent à Danaos la main de ses filles; leur père accepta, mais donna à ses filles des poignards et, pendant leur nuit de noce, elles tuèrent leurs maris, alors qu'ils dormaient. Seule Hypermnestre épargna son époux, Lynceus. Plus tard, les Danaïdes se marièrent une deuxième fois ${ }^{85}$.

Les Danaïdes, ces jeunes femmes-« loups », arrivées à Argos comme des étrangères, qui tuent leurs maris d'une manière perfide, et qui implorent la bienveillance d'Apollon Lykeios, font penser au "loup " Oreste, tel qu'il apparaît dans l'Électre de Sophocle : éphèbe guidé par Apollon Lykeios, qui se fait passer pour un étranger, et qui égorge ses victimes en recourant à la ruse. Si, dans la pièce de Sophocle, Oreste est présenté comme un jeune homme au cours de la phase de « marge » de son initiation, puis au moment de son intégration à la société des adultes, on peut admettre que les Danaïdes, telles qu'elles apparaissent dans les Suppliantes d'Eschyle et dans le récit d'Apollodore, sont également des jeunes filles traversant une période d'initiation, avant d'accéder au statut d'adulte. Leur refus du mariage, leur fuite devant les fils d'Égyptos et l'acte meurtrier qu'elles commettent avec la perfidie du loup représenteraient alors le comportement d'« initiants » pendant leur période de marge, période caractérisée par l'inversion des valeurs de la société et un comportement marqué par la sauvagerie. Cela correspond à ce qu'observe K. Dowden, dans son ouvrage sur les rites d'initiation réservés aux jeunes filles dans diverses régions de $\mathrm{Grèce}^{86}$, lorsqu'il évoque les Danaïdes : jeunes filles venues d'un pays étranger - trait qui « représente bien

\footnotetext{
84 WILAMOWITZ, $1955^{2}$ [1931-1932] (n. 4), p. 144.

85 Apollod., II, 1, 5; Paus., III, 12, 2; Pind., Pyth. 9, 111-116.

86 K. DowDen, Death and the Maiden, Girls Initiation Rites in Greek Mythology, London, New York, 1989, p. 147-165, ici 153-161. Voir aussi : R. SEAFord, The Tragic Wedding, in JHS, 107 (1987), p. 106-130 et, en dernier lieu : M. PIÉrart, Omissions et malentendus dans la "Périégèse »: Danaos et ses filles à Argos, in Kernos, Suppl. 8 (1998),
} p. $165-193$. 
l'aspect marginal et extérieur, si important dans la phase de marge des rites de passage ${ }^{87}$ - que leur refus du mariage pousse à fuir leurs prétendants, puis à les tuer, et qui, plus tard, se marient une deuxième fois, accédant ainsi finalement à leur situation normale de femme adulte.

Dans l'Électre de Sophocle, on a vu qu'Apollon Lykeios peut être considéré comme le dieu qui guide le "loup " Oreste au cours de son initiation. On peut donc faire l'hypothèse qu'Apollon Lykeios, dont les Danaïdes, comparées à des loups, implorent la bienveillance dans les Suppliantes, est aussi le dieu qui préside à leur initiation. Cependant, s'il joue un rôle pendant la période où elles fuient leurs prétendants, puis les tuent, il ne semble pas intervenir, selon les sources dont nous disposons, au moment de leur second mariage, c'est-à-dire au moment de leur intégration dans la sociétét ${ }^{88}$. Mais toutes les Danaïdes ne connurent pas le même sort. Hypermnestre, on l'a déjà vu, épargna son époux, Lynceus ${ }^{89}$. Selon Pausanias, elle fut jugée par les Argiens parce qu'elle avait désobéi à son père. Acquittée, elle consacra une statue d'Aphrodite Niképhoros dans le temple d'Apollon Lykeios. Puis, elle épousa Lynceus, qui devint roi d'Argos après Danaos ${ }^{90}$. Comme l'a observé $F$. Graf', on peut dire qu'Apollon Lykeios joue ainsi un rôle dans l'établissement sur le trône d'Argos du couple formé par Lynceus et Hypermnestre.

On estime, à la suite des travaux de F. Graf notamment, que le culte d'Apollon Lykeios a joué un rôle dans la vie politique de certaines cités grecques, dans le sens que le dieu peut être associé à l'idée d'un renouveau, d'un nouveau commencement, dans le domaine de la culture ou des institutions $^{92}$. Ainsi, à Argos, Apollon Lykeios est lié, selon Pausanias, à l'accession au pouvoir de Danaos, puisque, alors que Danaos revendiquait le pouvoir aux dépens du roi Gélanor, l'intervention d'un loup, attribuée à Apollon, décida les Argiens à lui accorder la royautée ${ }^{93}$. Or Danaos fut non seulement un nouveau roi, mais aussi le fondateur d'une nouvelle lignée royale, et l'on peut dire, selon F. Graf, qu' « avec Danaos commence pour la cité un nouvel ordre ». Lyncée et Hypermnestre furent le premier couple de cette lignée. Donc, Apollon Lykeios intervient dans l'établissement d'un nouvel ordre à Argos, d'une part en favorisant la prise de pouvoir de Danaos; d'autre part en

87 DowDEN, op. cit. (n. 86), p. 160.

$88 G$. supra, n. 85. On ignore cependant quelle situation prévalait dans la suite de la trilogie à laquelle appartenaient les Suppliantes d'Eschyle.

89 On n'abordera pas ici la légende d'Amymonè, qui, séduite par Poséidon ( $c f$. Apollod., II, 1, 4), ne participa pas, elle non plus, au meurtre collectif commis par ses sœurs.

90 Paus., II, 19, 6; Apollod, II, 1, 5 - II, 2, 1.

91 Graf, op. cit. (n. 1), p. 223.

92 GRAF, op. cit. (n. 1), p. 222-223; voir aussi BuRKert, 1975 (n. 41), p. 19-20; Musti, TORELLI, op. cit. (n. 40), p. 274.

93 Cf. $\$ 1$. 
contribuant à l'accession au trône de Lyncée et d'Hypermnestre. Par cette deuxième intervention, il consolide le nouvel ordre, qui avait été compromis au moment de la crise provoquée par le meurtre des fils d'Égyptos ${ }^{94}$.

Dans plusieurs des cités où son culte est attesté, Apollon Lykeios est lié d'une autre façon encore à un nouveau commencement dans le domaine de la vie civique: par son association avec l'institution de l'éphébie ${ }^{95}$. En effet, W. Burkert associe Apollon Lykeios aux assemblées au cours desquelles les éphèbes étaient reçus dans la communauté des hommes adultes ${ }^{96}$. M. Jameson a étudié une inscription athénienne $\mathrm{du} \mathrm{v}^{\mathrm{e}}$ siècle $\mathrm{e}^{97}$, trouvée à proximité $\mathrm{du}$ Lykeion, qui, comme l'indique Pausanias, était à l'origine un sanctuaire d'Apollon Lykeios ${ }^{98}$. M. Jameson estime que cette inscription met en rapport Apollon Lykeios avec l'institution de l'éphébie; selon lui, Apollon Lykeios était, à Athènes, « le dieu (...) des hoplites qui ont passé leurs tests et ont été complètement acceptés "; il intervenait au moment du passage à l'âge adulte, à la fin de l'éphébie ${ }^{99}$. F. Graf, passant en revue les différents lieux de culte d'Apollon Lykeios, a montré que le dieu était lié à l'éphébie, dans ses aspects militaires, et à travers le «nouveau commencement politique » que cette institution suppose $\mathrm{e}^{100}$.

Dans le domaine légendaire où évoluent Oreste et les Danaïdes, Apollon Lykeios, on a essayé de le montrer, peut être considéré comme un « dieu de l'initiation », qui guide les jeunes gens lors de leur passage de l'enfance au statut d'adulte. Cette interprétation s'accorde avec le rôle "politique » attribué à Apollon Lykeios. L'accession au pouvoir d'Oreste, membre de la famille des Atrides, rétablit à Argos l'ordre qui prévalait avant l'intervention de l'usurpateur Égisthe. L'installation de Lynceus et d'Hypermnestre sur le trône d'Argos consolide l'ordre établi par l'arrivée au pouvoir de Danaos. Mais, dans les deux cas, cet ordre n'est assuré qu'après l'intervention d'un acte meurtrier, commis avec ruse et sauvagerie par des « loups » qui agissent sous l'influence et avec la protection du dieu.

Au terme de cette étude, il est possible de dégager les traits qui semblent caractéristiques des interventions d'Apollon Lykeios dans la tragédie. D'une part, si l'on considère la manière dont il répond aux prières qui lui sont adressées, on découvre un dieu qui détruit, avec la perfidie du loup, le suppliant

94 Graf, op. cit. (n. 1), p. 223.

95 Contrairement à ce qui a été le cas jusqu'ici, le mot «éphébie » est maintenant pris dans son sens d'institution civique et militaire qui, dans plusieurs cités grecques, assurait le passage des jeunes gens de l'enfance à l'âge adulte, $C f$. n. 50.

96 W. BURKERT, Griechische Religion der archaischen und klassischen Epocbe, Stuttgart, 1977, p. 227.

97 M. Jameson, Apollo Lykeios in Athens, in Archaiognosia, 1 (1980), p. 213-236.

98 Paus., I, 19, 3.

99 JAMESON, loc. cit. (n. 97), p. 231-233.

100 Graf, op. cit. (n. 1), p. 225. 
qui se tourne vers lui. D'autre part, si l'on envisage Apollon Lykeios dans son rôle «politique », on le voit contribuer à établir un nouvel ordre ou à rétablir l'ordre dans la cité, mais après l'intervention d'un acte meurtrier sauvage et perfide, commis par des "loups » au service du dieu. Ainsi, dans les tragédies où il est mentionné, Apollon Lykeios se distingue par un mode d'intervention ambigu, où transparaissent les caractères prêtés à l'animal dont il porte le nom.

Département des Sciences de l'Antiquité

Claire-Françoise DE RoguIN

Université de Genève ${ }^{101}$

2 , rue de Candolle,

$\mathrm{CH}-1211$ GENÈVE 4

101 L'auteur remercie les Professeurs Claude Calame, de l'Université de Lausanne, Philippe Borgeaud et André Hurst, de l'Université de Genève, de leurs remarques, de leurs conseils et de l'intérêt qu'ils ont porté à ce travail. Elle est cependant entièrement responsable des opinions qui y sont exprimées. 